IL-2 dependent clones clearly indicate that both a CD3 + T cell population, and a distinct CD3- NK cell population mediate non-MHC restricted cytotoxicity ${ }^{2,3}$. There is no evidence to suggest that $\mathrm{CD} 3-\mathrm{NK}$ cells, a population comprising $\sim 10 \%$ of peripheral blood lymphocytes, are an immature member of the $\mathrm{T}$ cell lineage. Hopefully, the relationship of NK cells and $\mathrm{T}$ cells will be clarified by the discovery of the NK cell target recognition receptor.

LEWIS L. LANIER

Becton Dickinson Immunocytometry Systems,

PO Box 7375, Mountain View, California 94039, USA 1. Robertson, M. Nature 317, 768-771 (1985).
2. Ritz, J. et al. Science 228, 1540-1543 (1985).
3. Lanier, L.L.. Cwirla, S., Federspiel. N. \& Phillips, J.H. J.
exp. Med. (in the press).
4. Calman, A.F. \& Peterlin, B.M. Fed. Proc. 44, 1319 (1985). 4. Calman, A.F. \& Peterlin, B.M. Fed. Proc. 44, 1319 (1985).
Miranda RoBertson REPLIES-My remark on the possibility that NK cells might be arrested $\mathrm{T}$ cells was intended only as speculation: I was explicit about this. Ritz et al. ' did not address the question of rearrangement but state that truncated beta transcripts are made from T3- NK cells; Reynolds et al. (my ref. 34) state that beta genes are transcribed at low levels in LGL cells and "the size of the mRNA from the LGL leukaemias is restricted to be $1.0 \mathrm{~kb}$ size. A beta chain message of this size is probably transcribed from an unrearranged or simple DJ rearrangement" (sic). So it is true that I overstated the data in those two papers: in fact the only claim for rearrangement was by Yanagi et al. (Nature 314, 631; 1985; my ref. 33). I also accept that it does not necessarily follow from the data of Siliciano et al. (Nature 317, 428; 1985; my ref. 35) that NK cells belong to the $\mathrm{T}$ lineage, and my wording was incautious. Finally I heartily agree on the need for a precise definition of an NK cell and the identification of its receptor. $\square$

\section{The search for extraterrestrials}

SIR-I welcomed the review article by M.D. Papagiannis on the Search for extraterrestrial intelligence (SETI) (Nature 318, 13; 1985) which, I am sure, gives an accurate view of the global programme.

Faced with the cosmic haystack, I could not help thinking that the approach to SETI so far was somewhere between that of the drunk in the dark who searched for the lost diamond ring under the lamppost, and the rich father-in-law who brought in a bulldozer to do the job properly. The lamppost approach is to some extent inevitable: we have to search in spectral regimes that are technically open to us. But faced with a dauntingly wide spectrum there is opportunity to use a bit more intelligence and a bit less bulldozer when it comes to constructing vast capacity multichannel spectrum analysers.
First, the microwave window (Fig. 2 of Papagiannis) has a fairly well defined minimum arising from the cosmic background, the galactic non-thermal background and the quantum limit. This will be apparent to any galactic civilization capable of interstellar communications and occurs between 4 and $6 \mathrm{GHz}$.

Second, the so called magic frequencies of $\mathrm{H}$ and $\mathrm{OH}$, while they are most important as absolute standards, are contaminated by natural backgrounds and they do not occur at the optimum frequency in the microwave window.

What SETI needs is a "search filter" which it can apply to the cosmic haystack so as to assign search priorities on the basis of the rational choice of transmission frequency. This choice should combine a physical constraint such as the energy constraint which defines the minimum in the microwave window, an absolute frequency standard such as the $\mathrm{H}$ line, and a civilization signature constant (CSC): some absolute number by which the frequency standard can be multiplied or divided to obtain the final transmission frequency.

The most obvious choice for the CSC is $\pi$ or e, and using the $\mathrm{H}$ line we arrive at $4.468 \mathrm{GHz}$ and $3.863 \mathrm{GHz}$ as most likely communication frequencies. Since it is difficult to determine the cultural bias in choosing the CSC, care should be taken in investigating such CSCs as $(e \pi)^{4}, \pi^{2}$ and dimensionless physical constants.

If the systematic searches of the microwave spectrum first concentrate on a narrow frequency band that encompasses the Doppler broadening about the above absolutely defined frequencies, they will increase their chance of early success, without prejudicing the rest of the search.

Unimagined technologies could radically alter the physical constraints. The physical constraint would be quite different for a civilization which is in close proximity to an old and permanently nulled pulsar. In this case the pulsar could very effectively be used as a broad band transmitter of radio pulses, by firing projectiles at it with the appropriate modulation. Since a significant fraction of the projectile rest mass would be converted to radiation, this would provide a powerful means of interstellar communications, with a range of several kiloparsecs. It might be worth examining known pulsars for signs of amplitude or phase modulation.

Papagiannis is right that the answer can only come through "scientific searches ... pursued vigorously", but we should give "them" credit for some rationality when we devise the search. If they do not want to communicate, and if we are simply eavesdropping, the bulldozer approach may be the only way, but the chance of early success will then probably be limited.

D.G. BLAIR

Department of Physics,

Nedlands, Western Australia 6009

\section{Clone controversy at Immunex}

SIR-At the Fourth International Lymphokine Workshop in West Germany on 17-21 October 1984, an executive of the company Immunex challenged our claim to have cloned the complementary DNA for interleukin-1 (IL-1) and informed the workshop that we had instead cloned some other monocyte product produced in response to lipopolysaccharide stimulation. Although not willing to present any scientific data to validate his claim at the time, he then added that Immunex had obtained a similar clone to ours several months previously, but had discounted it as being that of IL-1. A full account of this public outburst is now available ${ }^{1}$.

We therefore read with great interest the recent article ${ }^{2}$ entitled "Cloning, sequence and expression of two distinct human interleukin-1 complementary DNAs" from Immunex Corporation, of which the same executive is an author. Immunex now obviously feels confident about the fidelity of our human IL-1 cDNA clone since they confirm our work on the molecular cloning of the predominant ( $\mathrm{pI}$ 7) form of monocyte-derived human interleukin-1 which is now published ${ }^{3}$.

In addition Immunex has added unnecessary confusion to the field by assigning an ' $\alpha-\beta$ ' nomenclature to the $\mathrm{IL}-1$ sequences. We take issue with this action on two counts. First, we feel that the establishment of such classification is premature when based solely on characterization of cDNA clones. A meaningful classification can only be established when information is available on complexities of genomic organization, post-transcriptional and/or translational processing and the relationship to the multiple forms of IL-1 protein which have been described. Second, in the event that the ' $\alpha-\beta$ ' system were to be adopted, we suggest that it would be more appropriate to call the pI 7 form ' $\alpha$ ' and the pI 5 ' $\beta$ ' rather than the reverse which was proposed by Immunex. This suggestion is based upon criteria of prevalence of the molecule (50-fold more pI 7 than pI 5) and the chronology of the cloning in the human.

\section{SHELdon M. WOLFF}

Philip E. Auron

Charles A. Dinarello

LANNY J. ROSENWASSER

New England Medical Center,

Boston, Massachusetts 02111, USA

Andrew C WebB

Wellesley College,

Wellesley, Massachusetts 02181, USA

Alexander Rich

Massachusetts Institute of Technology,

Cambridge Massachusetts 02139, USA

1. Lymphokine Res. 4. 51 (1985)

2. March. C.J. et al. Nature 315, 641-647 (1985).

3. Auron, P.E. et al Proc. natn. Acad. Sci. U.S.A. 81, 7907-791 (1984). 